\title{
Methods for Noise Reduction in Far-Field Patterns Obtained From Cylindrical Near-Field Antenna Measurements
}

\author{
F. J. Cano-Fácila and M. Sierra-Castañer
}

\begin{abstract}
Two different methods to reduce the noise power in the far-field pattern of an antenna as measured in cylindrical nearfield (CNF) are proposed. Both methods are based on the same principle: the data recorded in the CNF measurement, assumed to be corrupted by white Gaussian and space-stationary noise, are transformed into a new domain where it is possible to filter out a portion of noise. Those filtered data are then used to calculate a far-field pattern with less noise power than that one obtained from the measured data without applying any filtering. Statistical analyses are carried out to deduce the expressions of the signal-tonoise ratio improvement achieved with each method. Although the idea of the two alternatives is the same, there are important differences between them. The first one applies a modal filtering, requires an oversampling and improves the far-field pattern in all directions. The second method employs a spatial filtering on the antenna plane, does not require oversampling and the far-field pattern is only improved in the forward hemisphere. Several examples are presented using both simulated and measured near-field data to verify the effectiveness of the methods.
\end{abstract}

Index Terms-Additive white Gaussian noise (AWGN), antenna measurements, filtering, modal analysis, noise cancellation, reconstruction algorithms, signal-to-noise ratio.

\section{INTRODUCTION}

I $\mathrm{N}$ many cases, antenna radiation patterns and other farfield parameters such as gain, directivity, sidelobe level, beamwidth, etc., cannot be determined directly from measurements taken in a far-field range because the distance to the farfield region may be too long. However, it is well known that those parameters can be obtained using analytical transformations from near-field measurements [1]-[3]. In these cases, the radiation pattern is obtained by processing the near-field data. Therefore, an uncertainty analysis is required to assess the effect of near-field errors on the accuracy of the far-field pattern. References [4]-[6] are examples of studies that examine the relationships between the measurement errors and their effects on the far-field using mathematical analyses, simulations, or measurement tests.

This paper focuses on random near-field errors, which are always present and are commonly introduced by the receiver additive noise. In many cases, these errors are negligible in the overall measurement uncertainty thanks to the use of modern receivers and sufficient amplification in the system. However, when measuring ultra-low sidelobe or high-performance antennas, noise may significantly alter the radiation pattern and it has to be taken into account in the near-field error budget Some comprehensive studies for random noise in near-field measurements have already been presented. For the planar system, two independent analyses with similar results have been described in [7], [8]. Both of them start with random errors in planar near-field (PNF) and obtain expressions that represent the signal-to-noise ratio in far-field as a function of the noise power in near-field. A similar study for cylindrical near-field (CNF) measurements was carried out in [9], [10]

The objective of this paper is not to analyze the effect of random near-field errors in the far-field results, but to describe methods to improve the signal-to-noise ratio in the far-field pattern by reducing the noise power. This reduction is achieved by representing the acquired near-field data in a convenient domain where it is possible to filter out a portion of noise. This idea of reducing the noise by means of a filtering in an auxiliary domain was proposed in [11] and [12] for the spherical near-field (SNF) case. The same idea was used in [13] to improve the signal-to-noise ratio in the far-field pattern of an antenna as measured in PNF. In this last case, however, a spatial filtering on the antenna under test (AUT) plane is employed instead of a modal filtering. In the present work, two different methods to reduce the noise power when measuring an antenna in CNF are proposed. Moreover, a detailed statistical analysis is performed in each case in order to determine the signal-to-noise ratio improvement.

This paper is organized as follows. Section II describes a modal filtering method. In this section, a statistical analysis to determine the signal-to-noise ratio improvement achieved with this first filtering method as well as a study to obtain the relationship between that improvement and the required oversampling are carried out. In Section III, the second method based on a filtering on the AUT plane is presented. As in the previous section, the statistical properties of the noise in this new domain are determined in order to derive the expression for the signal-to-noise ratio improvement. Several results are shown in Section IV by using both simulated and measured near-field data. Conclusions are drawn in Section V.

\section{Modal Filtering Method}

The noise mitigation will be accomplished in both methods by means of filtering before obtaining the far-field results. Be- 
cause all the measured data are always noise corrupted, filtering cannot be applied to this initial information and an auxiliary data representation that allows a noise filtering without cancelling out desired information is needed.

As it is well-known, the initial step to determine the farfield pattern from CNF measurements is to calculate two sets of cylindrical modal coefficients (CMCs) [14].

As deduced from [15], the CMC are band-limited in the $n-k_{z}$ domain, being negligible outside of the visible region defined by

$$
n^{2}+\left(k_{z} a_{0}\right)^{2}<\left(k_{0} a_{0}\right)^{2}
$$

where $a_{0}$ is the radius of the smallest sphere enclosing the AUT.

According to (1), in order to avoid the aliasing error when calculating the $\mathrm{CMC}$, the maximum admissible sampling spacing in $\phi$ and $z$ is

$$
\begin{gathered}
2 k_{0} \leq\left.\frac{2 \pi}{\Delta z} \Rightarrow \Delta z\right|_{\max }=\frac{\lambda}{2} \\
2 k_{0} a_{0} \leq\left.\frac{2 \pi}{\Delta \phi} \Rightarrow \Delta \phi\right|_{\max }=\frac{\lambda}{2 a_{0}} .
\end{gathered}
$$

However, the validity region of the far-field pattern obtained from a CNF measurement is limited in elevation. Therefore, a sampling spacing in $z$ larger than a half wavelength is typically used.

If the separation between samples is smaller than the values indicated in (2), more CMC are obtained in the evanescent region, where theoretically have to be negligible. However, due to the presence of undesired contributions, like reflections, diffractions, leakage signals, etc., the calculated CMC may be nonzero outside of the visible region. Therefore, one alternative to cancel the unwanted effects of these contributions is to apply a modal filtering, setting all CMC in the evanescent region to zero. This idea of applying a modal filtering to suppress unwanted contributions and, in particular, reflections from the environment, was initially proposed for the CNF case in [16]. This last work was the extension of previous publications whose aim was the reflection suppression in the spherical case [17], [18] and most recently it has been applied in PNF [19] and far-field [20] measurements. A general strategy, which also exploits the band-limitation properties of the radiated field, to reduce the effect of the clutter noise, valid for arbitrary scanning and source geometries, was proposed in [21]. The first noise reduction method proposed in this paper is based on the principle presented in [16]. An oversampling is applied in order to obtain more CMC than required. After that, the evanescent modes are filtered out, removing the noise contribution in this region. Finally, the filtered CMC are used to calculate a far-field pattern with less noise power.

Once the main idea of this method has been described, a statistical analysis to determine the signal-to-noise ratio improvement is carried out. In the analysis, a complex white Gaussian and space-stationary noise is considered. Its mean and variance are assumed to be zero and $\sigma_{\text {nf }}^{2}$, respectively. Because expressions to calculate the modal coefficients [14] are linear, an independent analy sis can be performed for the AUT contribution and noise. As mentioned before, the CMC associated to the AUT contribution are theoretically zero outside of the region defined by (1). The noise contribution to the CMC can be determined by considering only the measured near-field white Gaussian noise in $\phi$ and $z$. Thus, the autocorrelation of the noise in each set of $\mathrm{CMC}$ is

$$
\begin{aligned}
& R_{a_{n}^{\mathrm{AWGN}}\left(k_{z}\right)}(\alpha, \beta) \\
& =E\left[a_{n+\alpha}^{\mathrm{AWGN}}\left(k_{z}+\beta\right) a_{n}^{\mathrm{AWGN}^{*}}\left(k_{z}\right)\right] \\
& =\frac{\Delta \phi^{2} \Delta z^{2}}{\left.\left(4 \pi^{2}\right)^{2}\left|\frac{\partial H_{n}^{(2)}}{\partial r}(\kappa r)\right|_{r=a}\right|^{2}} \\
& \times \sum_{l=1}^{N_{\phi}} \sum_{i=1}^{N_{z}}\left(\frac{n^{2} k_{z}^{2}}{a^{2} \kappa^{4}} E\left[\left|E_{z}\left(\phi_{l}, z_{i}\right)\right|^{2}\right] E\left[\left|E_{\phi}\left(\phi_{l}, z_{i}\right)\right|^{2}\right]\right) \\
& =\frac{\Delta \phi^{2} \Delta z^{2} N_{\phi} N_{z} \sigma_{\mathrm{nf}}^{2}}{\left.\left(4 \pi^{2}\right)^{2}\left|\frac{\partial H_{n}^{(2)}}{\partial r}(\kappa r)\right|_{r=a}\right|^{2}}\left(\frac{n^{2} k_{z}^{2}}{a^{2} \kappa^{4}}+1\right) \delta(\alpha, \beta) \\
& R_{b_{n}^{\mathrm{AWGN}}\left(k_{z}\right)}(\alpha, \beta) \\
& =E\left[b_{n+\alpha}^{\mathrm{AWGN}}\left(k_{z}+\beta\right) b_{n}^{\mathrm{AWGN}^{*}}\left(k_{z}\right)\right] \\
& =\frac{k_{0}^{2} \Delta \phi^{2} \Delta z^{2}}{\left(4 \pi^{2}\right)^{2}\left|H_{n}^{(2)}(\kappa a)\right|^{2} \kappa^{4}} \sum_{l=1}^{N_{\phi}} \sum_{i=1}^{N_{z}} E\left[\left|E_{\phi}\left(\phi_{l}, z_{i}\right)\right|^{2}\right] \\
& =\frac{k_{0}^{2} \Delta \phi^{2} \Delta z^{2} N_{\phi} N_{z} \sigma_{\mathrm{nf}}^{2}}{\left(4 \pi^{2}\right)^{2}\left|H_{n}^{(2)}(\kappa a)\right|^{2} \kappa^{4}} \delta(\alpha, \beta)
\end{aligned}
$$

where $\Delta \phi$ and $\Delta z$ are the azimuthal and vertical sampling spacing, respectively, $H_{n}^{(2)}$ is the Hankel function of second kind of order $n, k_{0}$ represents the free-space wavenumber, $a$ is the radius of the measurement cylinder, $N_{\phi}$ and $N_{z}$ stand for the number of samples along $\phi$ and $z$ coordinates, respectively, and $\kappa=\sqrt{k_{0}^{2}-k_{z}^{2}}$. The previous expressions are valid when using an ideal probe. In the case of arbitrary probes, the expressions are similar, except for the presence of corrective factors which depend on the probe receiving coefficients [22].

The CMC obtained considering only noise are Gaussian random variables because they are expressed as a sum of independent Gaussian random variables. Moreover, all of them have zero mean and a variance (autocorrelation for $\alpha=\beta=0$ ) that is directly proportional to the near-field noise variance. However, as deduced from (3) and (4), these CMC are nonstationary because the autocorrelation depends on $n$ and $k_{z}$. Nevertheless, the CMC are nonzero for all values of $n$ and $k_{z}$, and as commented before, a modal filtering can be applied to suppress the noise located in the evanescent region.

Before presenting the modal filtering, it is necessary to determine the characteristics of the noise in each far-field component in order to obtain a reference with which to compare the results after the filtering process. These far-field components are obtained as described in [14] and the autocorrelation of the noise in each far-field component is

$$
\begin{aligned}
& R_{E_{\phi}^{\mathrm{AWGN}}(\theta, \phi)}(\gamma, \mu) \\
& \quad=\left(2 k_{0} \sin (\theta)\right)^{2} \sum_{n=-N_{\phi} / 2}^{N_{\phi} / 2} R_{a_{n}^{\mathrm{AWGN}}\left(k_{z}\right)}(0,0) e^{j n \mu} \delta(\gamma)
\end{aligned}
$$




$$
\begin{aligned}
= & \frac{\Delta \phi^{2} \Delta z^{2} N_{\phi} N_{z} \sigma_{\mathrm{nf}}^{2}}{\left(2 \pi^{2}\right)^{2}} \\
& \times \sum_{n=-N_{\phi} / 2}^{N_{\phi} / 2} \frac{n^{2} k_{z}^{2}+a^{2} \kappa^{4}}{\left.\left.\frac{\partial H_{n}^{(2)}}{\partial r}(\kappa r)\right|_{r=a}\right|^{2} a^{2} \kappa^{2}} e^{j n \mu} \delta(\gamma) \\
R_{E_{\theta}^{\mathrm{AWGN}}(\theta, \phi)}(\gamma, \mu) & \\
= & \left(2 k_{0} \sin (\theta)\right)^{2} \sum_{n=-N_{\phi} / 2}^{N_{\phi} / 2} R_{b_{n}^{\mathrm{AWGN}}\left(k_{z}\right)}(0,0) e^{j n \mu} \delta(\gamma) \\
= & \frac{k_{0}^{2} \Delta \phi^{2} \Delta z^{2} N_{\phi} N_{z} \sigma_{\mathrm{nf}}^{2}}{\left(2 \pi^{2}\right)^{2} \kappa^{2}} \\
& \times \sum_{n=-N_{\phi} / 2}^{N_{\phi} / 2} \frac{1}{\left|H_{n}^{(2)}(\kappa a)\right|^{2}} e^{j n \mu} \delta(\gamma) .
\end{aligned}
$$

As deduced from (5) and (6) and as was stated in [9], the autocorrelation of the far-field noise depends on $\theta$ but not on $\phi$. Therefore, the noise is nonstationary in elevation and stationary in azimuth. Moreover, the noise is white in $\theta$ and colored in $\phi$ because the autocorrelation is zero for $\gamma \neq 0$ and nonzero for $\mu \neq 0$, respectively.

Up to now, a statistical study of the noise in the CNF to far-field transformation has been carried out. Although, this is not the main purpose of this work, the previous results provide useful information to achieve our objective, i.e., to be able to define a filtering to reduce the noise in the far-field pattern and to find the expression for the signal-to-noise ratio improvement.

From (3) and (4), it was deduced that the noise is not stationary but it is distributed over the whole $n-k_{z}$ domain. In contrast, the desired information of the AUT is concentrated in the region specified in (1). Therefore, a filtering as defined in (7) can be applied to remove a portion of noise without cancelling the CMC which contain the AUT information:

$$
F_{n}\left(k_{z}\right)= \begin{cases}1 & n^{2}+\left(k_{z} a_{0}\right)^{2}<\left(k_{0} a_{0}\right)^{2}(\text { visible }) \\ 0 & n^{2}+\left(k_{z} a_{0}\right)^{2} \geq\left(k_{0} a_{0}\right)^{2} \text { (evanescent) }\end{cases}
$$

This filtering is depicted in Fig. 1(a) and it is applied to the two sets of CMC. Once the evanescent modes has been filtered out, the noise in each far-field component is

$$
\begin{aligned}
& E_{\phi, \text { filt }}^{\mathrm{AWN}}(\theta, \phi) \\
& \quad=2 k_{0} \sin (\theta) \sum_{n=-N(\theta)}^{N(\theta)} j^{n} a_{n}^{\mathrm{AWGN}}\left(k_{0} \cos \theta\right) e^{j n \phi} \\
& E_{\theta, \text { filt }}^{\mathrm{AWGN}}(\theta, \phi) \\
& \quad=-j 2 k_{0} \sin (\theta) \sum_{n=-N(\theta)}^{N(\theta)} j^{n} b_{n}^{\mathrm{AWGN}}\left(k_{0} \cos \theta\right) e^{j n \phi}
\end{aligned}
$$

with

$$
\begin{aligned}
N(\theta) & =\sqrt{\left(k_{0} a_{0}\right)^{2}-\left(k_{z} a_{0}\right)^{2}} \\
& =k_{0} a_{0} \sqrt{1-\cos ^{2}(\theta)}=k_{0} a_{0} \sin (\theta) .
\end{aligned}
$$

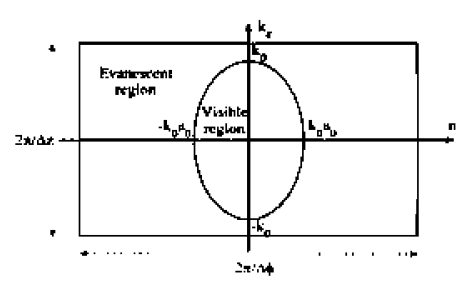

(a)

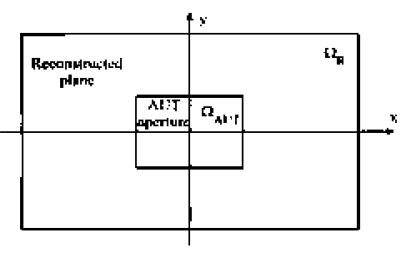

(b)

Fig. 1. Regions of interest used to define (a) the modal filtering and (b) the spatial filtering.

Therefore, the new autocorrelation of the far-field noise is

$$
\begin{gathered}
R_{E_{\phi, \text { filt }}^{\mathrm{AWGN}}(\theta, \phi)}(\gamma, \mu)=\frac{\Delta \phi^{2} \Delta z^{2} N_{\phi} N_{z} \sigma_{\mathrm{nf}}^{2}}{\left(2 \pi^{2}\right)^{2}} \\
\cdot \sum_{n=-N(\theta)}^{N(\theta)} \frac{n^{2} k_{z}^{2}+a^{2} \kappa^{4}}{\left.\left|\frac{\partial H_{r}^{(2)}}{\partial r}(\kappa r)\right|_{r=a}\right|^{2} a^{2} \kappa^{2}} e^{j n \mu} \delta(\gamma) \\
R_{E_{\theta, \mathrm{filt}}^{\mathrm{AWGN}}(\theta, \phi)}(\gamma, \mu)=\frac{k_{0}^{2} \Delta \phi^{2} \Delta z^{2} N_{\phi} N_{z} \sigma_{\mathrm{nf}}^{2}}{\left(2 \pi^{2}\right)^{2} \kappa^{2}} \\
\cdot \sum_{n=-N(\theta)}^{N(\theta)} \frac{1}{\left|H_{n}^{(2)}(\kappa a)\right|^{2}} e^{j n \mu} \delta(\gamma) .
\end{gathered}
$$

The far-field noise obtained after the filtering process is also a Gaussian noise with zero mean, uncorrelated and nonstationary in $\theta$, and correlated and stationary in $\phi$. When comparing (5) and (6) with (11) and (12) it is deduced that the only difference before and after applying the filtering is the number of terms in the summations. As deduced in (13), that number of terms is less or equal after the filtering stage.

Consequently, there is always a reduction of the noise power:

$$
N(\theta)=\frac{2 \pi}{\lambda} a_{0} \sin (\theta) \leq\left.\frac{N_{\phi}}{2}\right|_{\min }=\frac{\pi}{\left.\Delta \phi\right|_{\max }}=\frac{2 \pi}{\lambda} a_{0} .
$$

Moreover, the modal filtering does not modify the AUT contribution. Therefore, the signal-to-noise ratio improvement can be calculated as the ratio between the noise power before and after applying the filtering:

$$
\begin{aligned}
\Delta \operatorname{SNR}_{E_{\phi}}(\theta) & =\frac{R_{E_{\phi}^{\mathrm{AWGN}}(\theta, \phi)}(0,0)}{R_{E_{\phi, \text { filt }}^{\mathrm{AWGN}}(\theta, \phi)}(0,0)} \\
& =\frac{\sum_{n=-N_{\phi} / 2}^{N_{\phi} / 2} \frac{n^{2} k_{z}^{2}+a^{2} \kappa^{4}}{\left.\left.\frac{\partial H_{n}^{(2)}}{\partial r}(\kappa r)\right|_{r=a}\right|^{2} a^{2} \kappa^{2}}}{\sum_{n=-N(\theta)}^{N(\theta)} \frac{n^{2} k_{z}^{2}+a^{2} \kappa^{4}}{\left.\left|\frac{\partial H_{n}^{(2)}}{\partial r}(\kappa r)\right|_{r=a}\right|^{2} a^{2} \kappa^{2}}} \\
\Delta \operatorname{SNR}_{E_{\theta}}(\theta) & =\frac{R_{E_{\theta}^{\mathrm{AWGN}}(\theta, \phi)}(0,0)}{R_{E_{\theta, \text { filt }}^{\mathrm{AWGN}}(\theta, \phi)}(0,0)}
\end{aligned}
$$




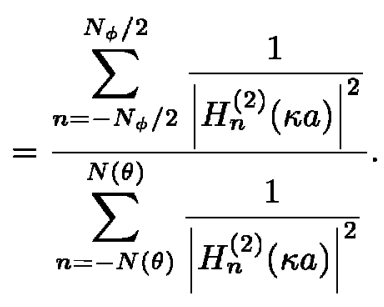

Several conclusions can be extracted from (14) and (15). First, a different signal-to-noise ratio improvement is obtained for each far-field component. Second, the improvement is dependent on $\theta$ but constant in $\phi$. Finally, the noise reduction depends on the number of measurement points in $\phi$, i.e., depends on the azimuthal sampling spacing but not on the sampling spacing in $z$. As deduced from (5) and (6), a larger oversampling in $z$ and $\phi$ implies a smaller far-field noise variance, and consequently, a better signal-to-noise ratio. However, the signal-to-noise ratio improvement introduced by the modal filtering depends only on the oversampling in $\phi$. In conclusion, an oversampling in both components introduces a signal-to-noise ratio improvement in the calculated far-field pattern, but when applying the modal filtering, a further noise reduction is achieved thanks to the oversampling in $\phi$.

Next, simpler approximate expressions for the improvement achieved with this first filtering method are derived. Assuming that the argument values of the Hankel function and the derivate of the Hankel function are very large, the asymptotic expansion of these functions can be used instead:

$$
\begin{gathered}
H_{n}^{(2)}(\kappa a) \approx j^{n+1 / 2}\left(\frac{2}{\pi \kappa a}\right)^{1 / 2} e^{-j \kappa a} \\
\left.\frac{\partial H_{n}^{(2)}}{\partial r}(\kappa a)\right|_{r=a} \approx-j^{n+3 / 2} \kappa\left(\frac{2}{\pi \kappa a}\right)^{1 / 2} e^{-j \kappa a}
\end{gathered}
$$

The latter expressions constitute a good approximation whenever $\kappa a \gg 1$. This condition is better fulfilled around $\theta=90^{\circ}$ where $\kappa \cong k_{0}$. Moreover, the validity region of a CNF measurement is also around the horizontal direction. Therefore, the better approximation is given for the region of interest. Thus, it follows that (14) and (15) reduce to

$$
\begin{aligned}
& \Delta \mathrm{SNR}_{E_{\phi}}(\theta) \cong \frac{\sum_{n=-N_{\phi} / 2}^{N_{\phi} / 2} \frac{n^{2} k_{z}^{2}+a^{2} \kappa^{4}}{\frac{2 \kappa^{4} a}{\pi \kappa}}}{\sum_{n=-N(\theta)}^{N(\theta)} \frac{n^{2} k_{z}^{2}+a^{2} \kappa^{4}}{\frac{2 \kappa^{4} a}{\pi \kappa}}} \cong \frac{N_{\phi}}{2 N(\theta)} \\
& \Delta \mathrm{SNR}_{E_{\theta}}(\theta) \cong \frac{\sum_{n=-N_{\phi} / 2}^{N_{\phi} / 2} \frac{\pi \kappa a}{2}}{\sum_{n=-N(\theta)}^{N(\theta)} \frac{\pi \kappa a}{2}}=\frac{N_{\phi}}{2 N(\theta)}
\end{aligned}
$$

where the following approximation has been taken into account for the $\phi$ component

$$
n^{2} k_{z}^{2}+a^{2} \kappa^{4} \cong a^{2} \kappa^{4} .
$$

Therefore, the improvement in both components is the same and is related to the sampling spacing in $\phi$ as follows:

$$
\begin{aligned}
\Delta \mathrm{SNR}_{\mathrm{fil}}(\theta) & \cong \frac{N_{\phi}}{2 N(\theta)}=\frac{\lambda}{2 \Delta \phi a_{0} \sin (\theta)} \\
& =\frac{\left.\Delta \phi\right|_{\max }}{\Delta \phi \sin (\theta)}=\frac{f_{\mathrm{ov}, \phi}}{\sin (\theta)}
\end{aligned}
$$

where it is deduced that the improvement is directly proportional to $f_{\mathrm{ov}, \phi}$, which is the oversampling factor in azimuth, and increases when we move away from the horizontal direction. Because the improvement is not a constant factor, the average signal-to-noise ratio improvement in the validity region can be considered as the figure of merit of this first filtering method:

$$
\begin{aligned}
\Delta \mathrm{SNR}_{\mathrm{AV}, \text { modal }} & =\frac{1}{2 \theta_{v}} \int_{\pi / 2-\theta_{v}}^{\pi / 2+\theta_{v}} \frac{f_{\mathrm{ov}, \phi}}{\sin (\theta)} d \theta \\
& =\frac{f_{\mathrm{ov}, \phi}}{2 \theta_{v}} \ln \left|\frac{1+\sin \left(\theta_{v}\right)}{1-\sin \left(\theta_{v}\right)}\right|
\end{aligned}
$$

where $\theta_{v}$ is the maximum validity angle measured from the horizontal direction.

Up to now, the signal-to-noise ratio improvement due to filtering has been evaluated with respect to the unfiltered case with the same sampling spacing. However, when comparing with the case without oversampling and without filtering, the improvement is as follows:

$$
\begin{aligned}
\Delta \mathrm{SNR}_{\mathrm{ov}-\mathrm{fil}}(\theta) \\
\quad \cong \frac{\Delta \phi_{\max }^{2} \Delta z_{\max }^{2} N_{\phi, \min } N_{z, \min }}{\Delta \phi^{2} \Delta z^{2} N_{\phi} N_{z}} \frac{N_{\phi, \min }}{2 N(\theta)} \\
\quad=f_{\mathrm{ov}, \phi} f_{\mathrm{ov}, z} \frac{2 \pi / \Delta \phi_{\max }}{2 k_{0} a_{0} \sin (\theta)} \\
\quad=f_{\mathrm{ov}, z} \frac{f_{\mathrm{ov}, \phi}}{\sin (\theta)}=\Delta \mathrm{SNR}_{\mathrm{ov}} \cdot \Delta \mathrm{SNR}_{\mathrm{fil}}(\theta)
\end{aligned}
$$

where $f_{\mathrm{ov}, z}$ is the oversampling factor in $z$ and $\Delta \mathrm{SNR}_{\mathrm{ov}}$ represents the improvement due to the oversampling, which is only dependent on the oversampling in $z$, i.e., there is an improvement due to the oversampling whenever the oversampling is performed in $z$ and there is an improvement due to the filtering whenever an oversampling in $\phi$ is applied.

Finally, the main steps of this first filtering method are summarized. A schematic of the method is depicted in Fig. 2. As commented before, the CNF data are used to obtain the CMC. This data representation allows filtering out a portion of noise without canceling AUT information. Once the modal filtering has been applied, a far-field pattern with less noise power is obtained.

\section{SPATIAl Filtering MethoD}

Another method to reduce the noise power in the far-field pattern obtained from a CNF measurement is presented in this section. As in the previous method, a field transformation that allows to filter out a portion of noise without modifying the desired signal is required. The transformation used here was already proposed in [13] for noise reduction in far-field results 


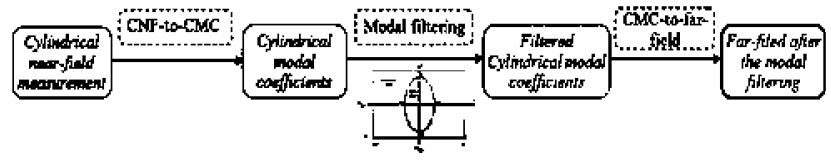

Fig. 2. Schematic of the modal filtering method.

obtained from PNF measurements and consists of back-propagating the measured field from the scan surface to the plane of the AUT. In this new domain, the desired contribution is theoretically concentrated in the antenna aperture, whereas the noise is spread over the whole reconstructed surface. Thanks to this fact, a spatial filtering may be applied, canceling the field located out of the AUT dimensions, which is only composed of noise.

As in the modal filtering method, a statistical analysis of the noise in this transformation is performed. Using acquired data containing only noise with the aforementioned statistical characteristics and applying a CNF-to-far-field transformation, the noise obtained in each far-field component is a complex Gaussian noise, with zero mean and autocorrelation given by (5) and (6). Once, the far-field components are known, an easy way to obtain the field distribution over the AUT plane is to calculate previously the plane wave spectrum (PWS), whose components are related to the extreme near-field of an antenna located in the $x y$-plane as indicated in (24):

$$
\begin{aligned}
& E_{\text {extreme }, \boldsymbol{x}-\boldsymbol{y}}(x, y) \\
& \quad=\int_{-\infty}^{\infty} \int_{-\infty}^{\infty} P_{x-y}\left(k_{x}, k_{y}\right) e^{-j k_{x} x} e^{-j k_{y} y} d k_{x} d k_{y} .
\end{aligned}
$$

Because in a CNF measurement the AUT is selected to be located in the $y z$-plane, the following change of coordinates is required before applying (24)

$$
x=y_{m} ; \quad y=z_{m} ; \quad z=x_{m}
$$

where $\left(x_{m}, y_{m}, z_{m}\right)$ are the measurement coordinates and $(x, y, z)$ represent the new coordinates used to calculate the extreme near-field. Therefore, the $x$ - and $y$-far-field components in the new coordinate system are

$$
\begin{aligned}
E_{x}\left(\theta_{m}, \phi_{m}\right)= & E_{y_{m}}\left(\theta_{m}, \phi_{m}\right) \\
= & E_{\theta_{m}}\left(\theta_{m}, \phi_{m}\right) \cos \left(\theta_{m}\right) \sin \left(\phi_{m}\right) \\
& \quad+E_{\phi_{m}} \cos \left(\phi_{m}\right) \\
E_{y}\left(\theta_{m}, \phi_{m}\right)= & E_{z_{m}}\left(\theta_{m}, \phi_{m}\right) \\
= & -E_{\theta_{m}}\left(\theta_{m}, \phi_{m}\right) \sin \left(\theta_{m}\right)
\end{aligned}
$$

where $\left(\theta_{m}, \phi_{m}\right)$ are the angular coordinates referenced to the measurement coordinate system, in which the far-field pattern is known and $E_{x_{m}}, E_{y_{m}}, E_{\theta_{m}}$ and $E_{\phi_{m}}$ are the $x-, y$-, $\theta$ - and $\phi$-far-field components in the measurement coordinate system, respectively.
Finally, the PWS components needed in (24) can be calculated as follows [1]:

$$
P_{x-y}\left(\theta_{m}, \phi_{m}\right)=\frac{E_{x-y}\left(\theta_{m}, \phi_{m}\right)}{j k_{0} \cos (\theta)}
$$

where $\theta$ represents the elevation angle in the new coordinate system. Therefore, the autocorrelation of the noise in each PWS component is

$$
\begin{aligned}
& R_{P_{x}^{\mathrm{AWGN}}\left(\theta_{m}, \phi_{m}\right)}(\varsigma, \tau) \\
& \quad=R_{E_{\theta}^{\mathrm{AWGN}}\left(\theta_{m}, \phi_{m}\right)}(\varsigma, \tau) \frac{\cos ^{2}\left(\theta_{m}\right) \sin ^{2}\left(\phi_{m}\right)}{k_{0}^{2} \cos ^{2}(\theta)} \\
& \quad+R_{E_{\phi}^{\mathrm{AWGN}}\left(\theta_{m}, \phi_{m}\right)}(\varsigma, \tau) \frac{\cos ^{2}\left(\phi_{m}\right)}{k_{0}^{2} \cos ^{2}(\theta)} \\
& R_{P_{y}^{\mathrm{AWGN}}\left(\theta_{m}, \phi_{m}\right)}(\varsigma, \tau) \\
& =R_{E_{\theta}^{\mathrm{AWGN}}\left(\theta_{m}, \phi_{m}\right)}(\varsigma, \tau) \frac{\sin ^{2}\left(\theta_{m}\right)}{k_{0}^{2} \cos ^{2}(\theta)}
\end{aligned}
$$

From the two previous expressions, it is deduced that the noise in the PWS components is nonstationary both in $\theta_{m}$ and $\phi_{m}$. Moreover, it is uncorrelated in $\theta_{m}$ and correlated in $\phi_{m}$. However, that correlation in azimuth can be considered almost negligible. Thanks to this fact, the determination of the noise characteristics in the extreme near-field is simplified in a great way. The $x$ - and $y$-components of the noise in the extreme near-field are calculated using the discrete versions of (24):

$$
\begin{aligned}
& E_{\text {extreme }, x-y}^{\mathrm{AWGN}}(x, y) \\
& =\sum_{a=1}^{N_{k_{x}}} \sum_{b=1}^{N_{k_{y}}} P_{x-y}^{\mathrm{AWGN}}\left(k_{x_{a}}, k_{y_{b}}\right) e^{-j k_{x_{a}} x} e^{-j k_{y_{b}} y}
\end{aligned}
$$

where $N_{k_{x}}$ and $N_{k_{y}}$ are the number of samples of the PWS taken on a regular $k_{x}-k_{y}$ grid. Because the PWS samples are known on a regular $\theta_{m}-\phi_{m}$ grid, and interpolation process is required. This interpolation introduces a correlation among samples, but as will be seen in Section IV, under certain conditions it is negligible and we can still assume that the noise is uncorrelated. Therefore, the autocorrelation of the noise in the extreme near-field can be easily calculated as shown (32):

$$
\begin{aligned}
& R_{E_{\text {extreme } x-y}^{\mathrm{AWGN}}(x, y)}(\chi, \xi) \\
&=\sum_{a 1=1}^{N_{k_{x}}} \sum_{b 1=1}^{N_{k_{y}}} \sum_{a 2=1}^{N_{k_{x}}} \sum_{b 2=1}^{N_{k_{y}}} E \\
& \quad \times\left[P_{x-y}\left(k_{x_{a 1}}, k_{y_{b 1}}\right) P_{x-y}^{*}\left(k_{x_{a 2}}, k_{y_{b 2}}\right)\right] \\
& \quad \cdot e^{-j k_{x_{a 1}}(x+\chi)} e^{-j k_{y_{b 1}}(y+\xi)} e^{j k_{x_{a 2}} x} e^{j k_{y_{b 2}} y} \\
&=\sum_{a=1}^{N_{k_{x}}} \sum_{b=1}^{N_{k_{y}}} R_{P_{x-y}^{\mathrm{AWGN}}\left(k_{x_{a}}, k_{y_{b}}\right.}(0,0) \\
& \cdot e^{-j k_{x_{a}} \chi} e^{-j k_{y_{b}} \xi} .
\end{aligned}
$$


From the previous expressions, it is deduced that the noise in the extreme near-field is correlated both in $\boldsymbol{x}$ and $\boldsymbol{y}$. However, all the samples have the same noise power (autocorrelation for $\chi=\xi=0$ ), i.e., the noise is stationary and is identically distributed over the whole reconstructed surface, whereas the desired field is theoretically concentrated in the aperture antenna. Therefore, a spatial filtering may be applied, canceling the field that is located out of the AUT dimensions and that is only composed of noise. This spatial filtering is defined in (33):

$$
G(x, y)= \begin{cases}1 & \Omega_{\mathrm{AUT}} \\ 0 & \Omega_{R}-\Omega_{\mathrm{AUT}}\end{cases}
$$

where $\Omega_{\boldsymbol{R}}$ and $\boldsymbol{\Omega}_{\mathrm{AUT}}$ represent the reconstructed plane and the AUT aperture depicted in Fig. 1(b).

Once the filtering has been described, an analysis to determine the signal-to-noise ratio improvement is carried out. Because the PWS and the extreme near-field are related by Fast Fourier Transforms, the Parseval's identity can be used to establish a relationship between the noise powers in both domains. The relationship before applying the spatial filtering is given by (34):

$$
\begin{aligned}
& \sum_{a=1}^{N_{x}} \sum_{b=1}^{N_{y}} R_{P_{x-y}^{\mathrm{AWGN}}\left(k_{x_{a}}, k_{y_{b}}\right)}(0,0) \\
&=\frac{1}{N_{x} N_{y}} \sum_{a=1}^{N_{x}} \sum_{b=1}^{N_{y}} R_{E_{\text {extreme }, x-y}^{\mathrm{AWGN}}\left(x_{a}, y_{b}\right)}(0,0)
\end{aligned}
$$

where $N_{x}$ and $N_{y}$ are the number of samples in the reconstructed plane, which are equal to the number of samples in the PWS, i.e., $N_{x}=N_{k_{x}}$ and $N_{y}=N_{k_{y}}$

After applying the filtering, the relationship is as follows:

$$
\begin{aligned}
& \sum_{a=1}^{N_{x}} \sum_{b=1}^{N_{y}} R_{P_{x-y, \text { filt }}^{\mathrm{AWGN}}\left(k_{x_{a}}, k_{y_{b}}\right)}(0,0) \\
& =\frac{1}{N_{x} N_{y}} \sum_{a=1}^{N_{x}} \sum_{b=1}^{N_{y}} G\left(x_{a}, y_{b}\right) R_{E_{\text {extreme }, x-y}^{\mathrm{AWGN}}\left(x_{a}, y_{b}\right)}(0,0) \\
& \quad=\frac{1}{N_{x} N_{y}} \sum_{a=1}^{N_{x}^{\prime}} \sum_{b=1}^{N_{y}^{\prime}} R_{E_{\text {extreme, }-y}^{\mathrm{AWGN}}\left(x_{a}, y_{b}\right)}(0,0)
\end{aligned}
$$

where $R_{P_{x-y, \text { filt }}^{\text {AwGN }}\left(k_{x_{a}}, k_{y_{b}}\right)}$ is the autocorrelation of the noise in the PWS after the noise filtering and $N_{x}^{\prime}$ and $N_{y}^{\prime}$ represent the number of samples within the AUT aperture. As deduced in (32), all the samples in the extreme near-field have the same noise power. Therefore, the last equation can be simplified as (36) indicates:

$$
\begin{aligned}
\sum_{a=1}^{N_{x}} \sum_{b=1}^{N_{y}} R_{P_{x-y, \text { filt }}^{\mathrm{AWGN}}\left(k_{x_{a}}, k_{y_{b}}\right)}(0,0) \\
=\frac{N_{x}^{\prime} N_{y}^{\prime}}{N_{x} N_{y}} \sum_{a=1}^{N_{x}} \sum_{b=1}^{N_{y}} R_{P_{x-y}^{\mathrm{AWGN}}\left(k_{x_{a}}, k_{y_{b}}\right)}(0,0) .
\end{aligned}
$$

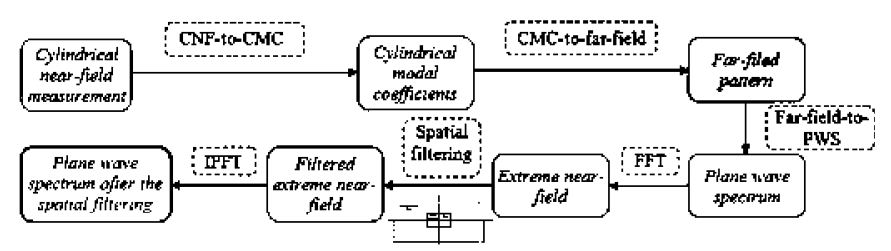

Fig. 3. Schematic of the spatial filtering method.

If now, (36) is divided by the total number of samples in both domains, the following result is obtained:

$$
\begin{aligned}
& \frac{1}{N_{x} N_{y}} \sum_{a=1}^{N_{x}} \sum_{b=1}^{N_{y}} R_{P_{x-y, f i t t}^{\mathrm{AWGN}}\left(k_{x_{a}}, k_{y_{b}}\right)}(0,0) \\
& =\frac{N_{x}^{\prime} N_{y}^{\prime}}{\left(N_{x} N_{y}\right)^{2}} \sum_{a=1}^{N_{x}} \sum_{b=1}^{N_{y}} R_{P_{x-y}^{\mathrm{AWGN}}\left(k_{x_{a}}, k_{y_{b}}\right)}(0,0) \\
& \Rightarrow P_{\mathrm{AV}, \text { filt }}=\frac{N_{x}^{\prime} N_{y}^{\prime}}{N_{x} N_{y}} P_{\mathrm{AV}}
\end{aligned}
$$

where $\boldsymbol{P}_{\mathrm{AV} \text {,filt }}$ and $\boldsymbol{P}_{\mathrm{AV}}$ are the average noise power in the PWS after and before applying the spatial filtering, respectively.

Because the spatial filtering does not modify the desired information (located within the AUT aperture), the last result can be employed to determine the average signal-to-noise ratio improvement achieved with this second method:

$$
\Delta \mathrm{SNR}_{\mathrm{AV}, \text { spatial }}=\frac{P_{\mathrm{AV}}}{P_{\mathrm{AV}, \mathrm{filt}}}=\frac{N_{x} N_{y}}{N_{x}^{\prime} N_{y}^{\prime}}=\frac{S_{\Omega_{R}}}{S_{\Omega_{\mathrm{AUT}}}}
$$

where $S_{\Omega_{R}}$ and $S_{\Omega_{\mathrm{AUT}}}$ symbolize the area of the reconstructed plane and AUT aperture, respectively. Although it seems we can obtained an infinite improvement by increasing the size of the reconstructed surface, there is a limitation that is related to the interpolation required in the process. A larger reconstructed surface implies a larger interpolation. Therefore, the PWS samples are more correlated and the previous samples in each measurement ring, i.e., the separation among analy sis is no longer valid. This effect is analyzed in detail in Section IV.

All the steps of this second filtering method are depicted in Fig. 3. The CNF data are used to obtain the far-field pattern by employing a modal expansion method. After that, the PWS components are calculated. Next, the extreme near-field is computed by means of a fast Fourier transform of the PWS. In this point, the spatial filtering defined in (33) is applied. Finally, a new PWS with less noise power is obtained by using an inverse fast Fourier transform.

\section{Numerical Results}

This section verifies the effectiveness of the proposed methods. The verification is carried out independently for each method by using both simulated and measured data.

\section{A. Modal Filtering Method}

The modal filtering method was firstly validated taking as input data the values of a simulation of a cylindrical near-field 


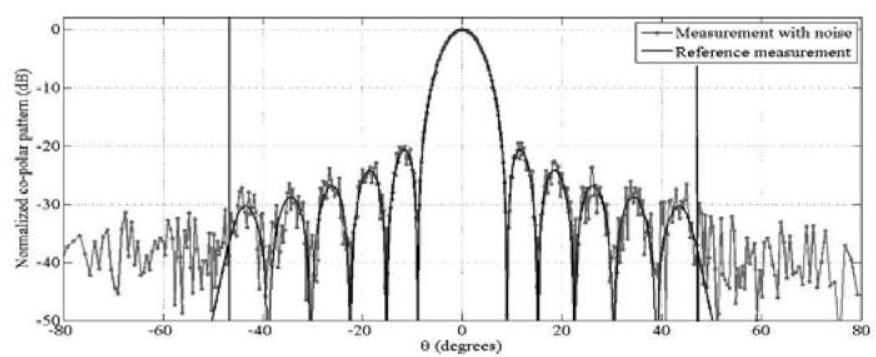

(a)

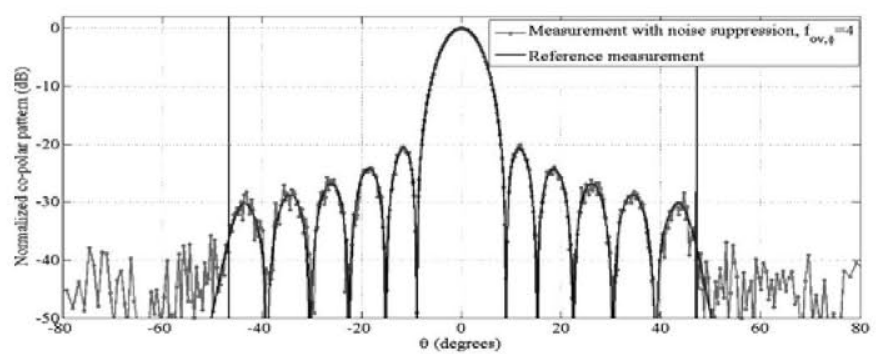

(b)

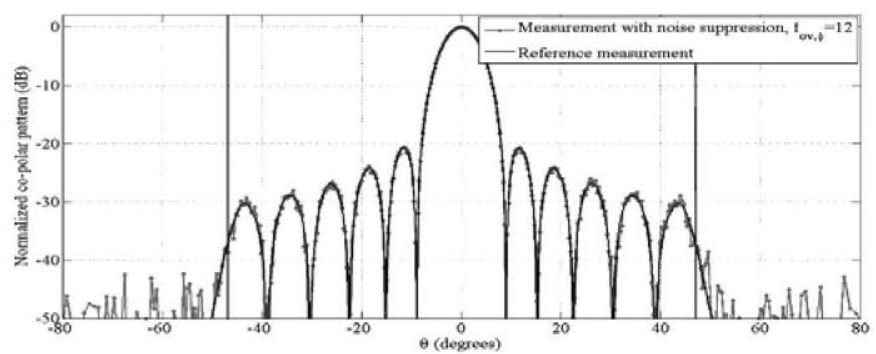

(c)

Fig. 4. Far-field comparison between (a) the reference pattern and the pattern with noise, (b) the reference pattern and the pattern after modal filtering with an oversampling factor equal to 4 and (c) the reference pattern and the pattern after modal filtering with an oversampling factor equal to 12 for the $\phi=0^{\circ}$ cut.

acquisition. The AUT used in the simulation was a $8 \lambda \times 8 \lambda$ aperture with a Gaussian-tapered field distribution. The frequency was $20 \mathrm{GHz}$, the samples were taken over a cylindrical surface of $3.38 \mathrm{~m}$ height $(225 \lambda)$, the radius of the cylinder was 1.5 $\mathrm{m}$ and the samples were spaced at $0.5 \lambda$ intervals in $\mathrm{z}$. Therefore, two simulations with a different sampling spacing in $\phi$ were performed. The first one took 288 samples was $1.25^{\circ}$. The second simulation recorded 900 samples per ring, i.e., the sampling spacing in azimuth was equal to $0.4^{\circ}$. Because the maximum sampling spacing is $5.06^{\circ}$, the oversampling factor was approximately 4 and 12, respectively. Moreover, Gaussian noise with $38 \mathrm{~dB}$ less power than the maximum of the simulated data was added in both simulations. Next, the modal filtering method was applied. The far-field pattern without noise suppression as well as the far-field patterns obtained after the noise filtering are compared with the reference far-field pattern in Fig. 4. As deduced in the theoretical analysis, the noise is more effectively reduced when the oversampling is greater. Fig. 5 shows the average signal-to-noise ratio improvement defined in (22) as a function of the maximum validity angle and for different oversampling factors. In our particular cases, where the maximum validity angle is $47.3^{\circ}$, the improvement is $6.58 \mathrm{~dB}$ for an oversampling of 4 and $11.35 \mathrm{~dB}$ for an oversampling of 12. Fig. 6

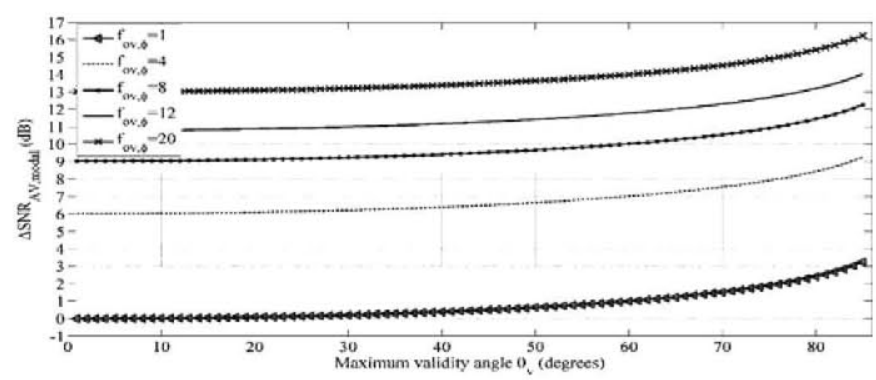

Fig. 5. Average signal-to-noise ratio improvement as a function of the maximum validity angle and for different oversampling factors.

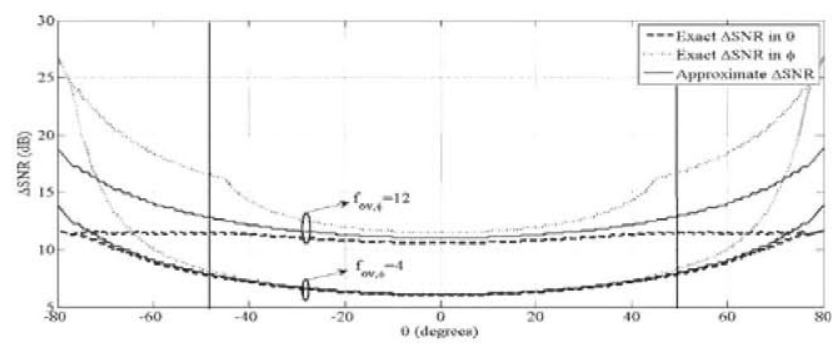

Fig. 6. Exact and approximate signal-to-noise ratio improvement as a function of the elevation angle for an oversampling factor equal to 4 and 12 .

depicts the exact improvement for both components calculated by using the expressions (14) and (15) and the improvement determined from the approximate expression given in (21) as a function of the elevation angle. Several conclusions can be extracted from Fig. 6. First, the improvement is not constant in elevation and increases when we move away from the horizontal plane. Second, the expression defined in (21) gives a better approximation around the mentioned plane, which is the region of interest. Finally, the approximation is better for small values of the oversampling factor.

The second validation uses information from an actual measurement in the cylindrical near-field range of the Technical University of Madrid (UPM). For the experiment, the probe and the AUT consisted of a corrugated conical-horn antenna and a $\mathrm{Ku}$-band reflector $(14 \mathrm{GHz})$, with a $40 \mathrm{~cm}$ diameter, respectively. The data were acquired over a cylinder with a height of $1.8 \mathrm{~m}$ and a radius of $0.63 \mathrm{~m}$ and with a spatial sampling equal to $0.5 \lambda$ in the vertical direction and $0.375^{\circ}$ in azimuth. Because the maximum sampling spacing in azimuth is $3.06^{\circ}$, the oversampling factor was approximately 8 . After measuring the AUT, Gaussian noise with $42 \mathrm{~dB}$ less power than the maximum of the acquired data was computationally added. The noise power was chosen to be large, so as to ensure a negligible measurement noise. The far-field obtained from the measured data without additive noise can thus be used as a reference to compare results before and after noise filtering. The far-field results obtained before and after applying the filtering are compared with the reference pattern in Fig. 7. According to (22) and Fig. 5, the average improvement was $9.61 \mathrm{~dB}$.

\section{B. Spatial Filtering Method}

The second method was also validated by using the simulation described in the modal filtering method. However, in this 


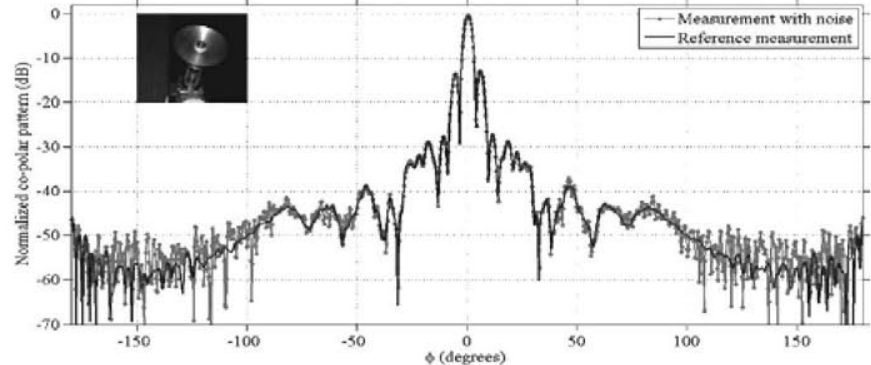

(a)

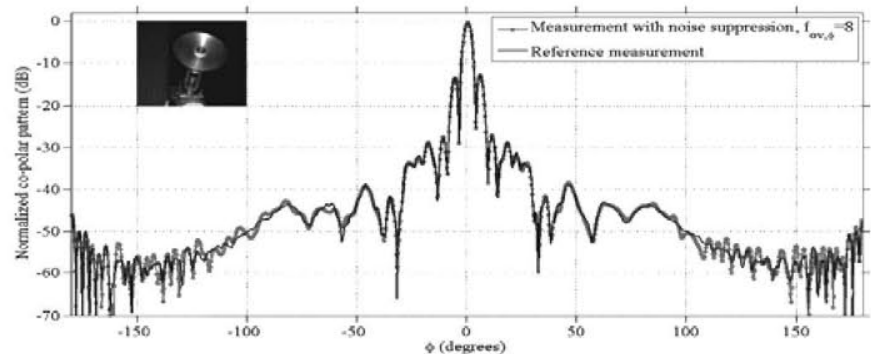

(b)

Fig. 7. Far-field comparison between (a) the reference pattern and the pattern with noise and (b) the reference pattern and the pattern after modal filtering with an oversampling factor equal to 8 in the horizontal plane.

second method, an oversampling in azimuth is not required, as deduced in the theoretical analysis. Therefore, the sampling spacing in azimuth used in the simulation is equal to the maximum sampling spacing, i.e., $3^{\circ}$. Moreover, Gaussian noise with $30 \mathrm{~dB}$ less power than the maximum of the simulated data was added, instead of a noise with $38 \mathrm{~dB}$ less power. The objective of increasing the noise power is to demonstrate that with this second method is possible to obtain a larger improvement using even a less number of acquired data. Once the new cylindrical acquisition was simulated, the extreme near-field was determined over a square plane of $225 \lambda$ side, which was equal to the height of the measurement cylinder. After that, a spatial filtering was applied, canceling the field that was located out of the AUT dimensions and that was only composed of noise. Finally, the filtered field distribution was inverse Fourier transformed back to the spectral domain, obtaining a new farfield pattern where the noise power was greatly reduced, as observed in Fig. 8. The average signal-to-noise ratio improvement achieved in this case was, according to (38), $28.98 \mathrm{~dB}$. Theoretically, with this method we can always increase the signal-tonoise ratio by using a larger reconstructed surface. However, this statement is not completely true because of the interpolation required in the spectral domain. A larger reconstructed surface implies a smaller sampling spacing in the PWS, i.e., more samples have to be interpolated from the known samples. Therefore, the correlation among noise samples increases and the assumption employed in (32) is no longer valid. As a result, the noise in the extreme near-field is nonstationary, decreasing from the center to the edges of the reconstructed plane. Consequently, the noise reduction in the filtering process is not as effective as when the noise is stationary. This effect is depicted in Fig. 9, where the theoretical improvement defined in (38) is compared with the improvement observed in different examples with different

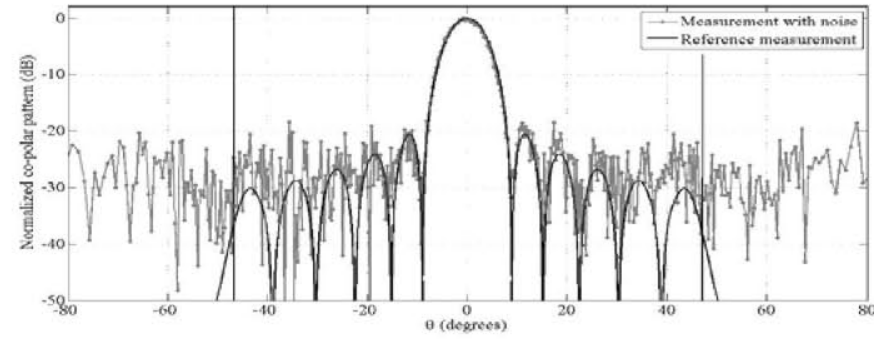

(a)

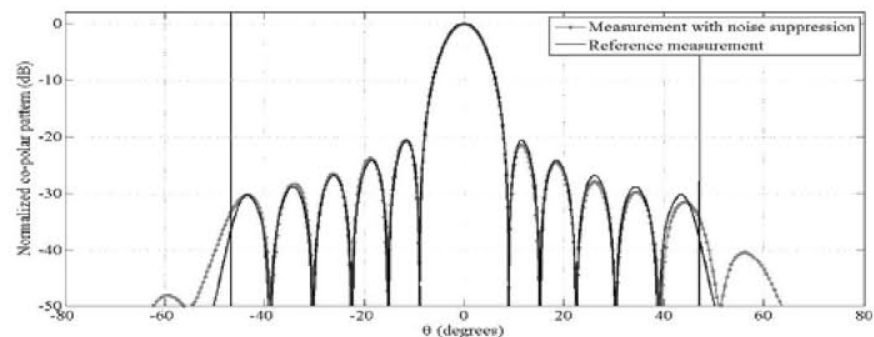

(b)

Fig. 8. Far-field comparison between (a) the reference pattern and the pattern with noise, (b) the reference pattern and the pattern after spatial filtering for the $\phi=0^{\circ}$ cut.

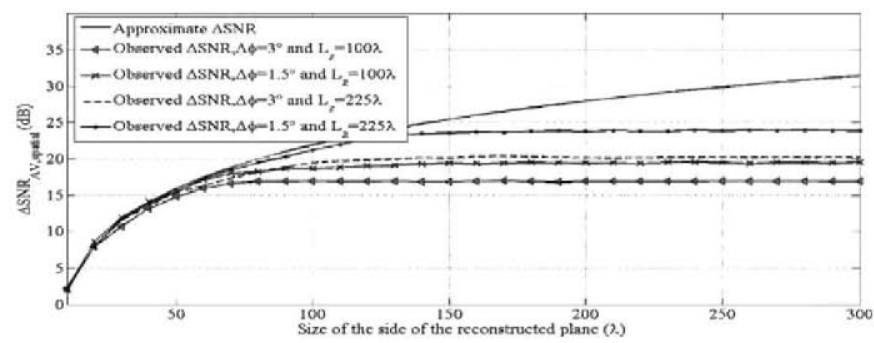

Fig. 9. Theoretical and observed average signal-to-noise ratio improvement for different acquisition parameters as a function of the size of the reconstructed surface.

acquisition parameters. As deduced from Fig. 9, when reducing the spatial sampling in $\phi$ or when increasing the height of the measurement cylinder, the improvement is larger for the same size of the reconstructed surface because, in both cases, we are reducing the spacing among known samples in the PWS, and a less number of samples have to be interpolated. Moreover, once the size of the reconstructed surface is larger than the height of the cylinder, the improvement remains constant. Due to this effect, the improvement is not $28.99 \mathrm{~dB}$ but $20.20 \mathrm{~dB}$, which is the maximum achievable with a spatial sampling in $\phi$ equal to $3^{\circ}$ and a height of the measurement cylinder equal to $225 \lambda$.

Finally, the spatial filtering method was validated by employing the data from the actual measurement described before. The only changes were, as in the previous example, the noise power, that in this case was $35 \mathrm{~dB}$ less than the maximum of the measured data, and the sampling spacing in $\phi$, that was equal to $1.5^{\circ}$. The reconstructed surface employed in this case was a $1.8 \mathrm{~m} \times 1.8 \mathrm{~m}$ plane. Because the AUT was a reflector of $0.4 \mathrm{~m}$ diameter, the theoretical improvement was $14.11 \mathrm{~dB}$, but due to the interpolation process, the observed improvement was $13.29 \mathrm{~dB}$. Up to now, the desired field has been assumed 


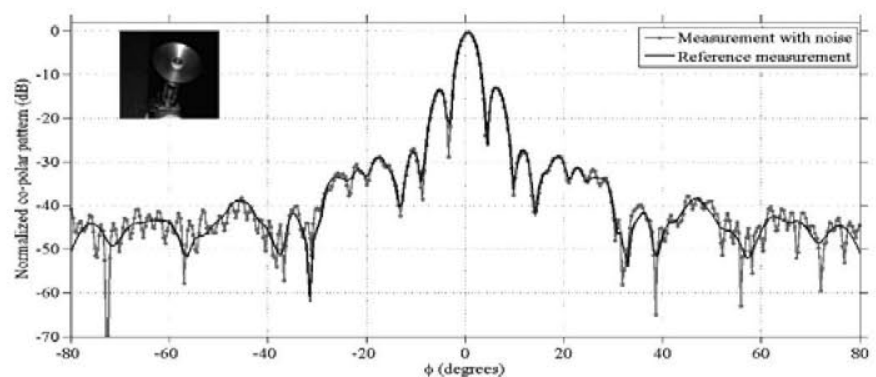

(a)

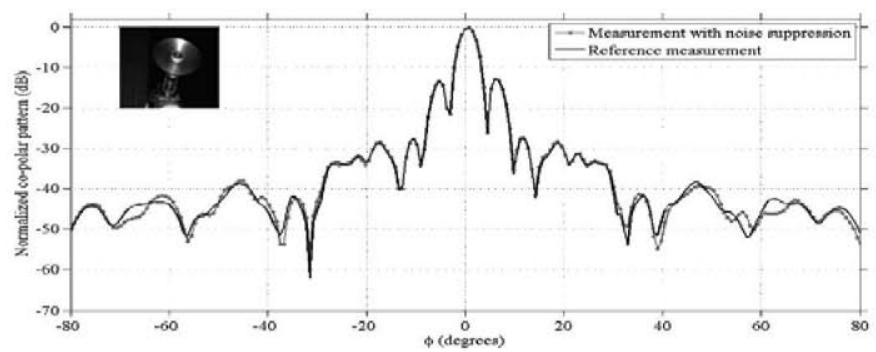

(b)

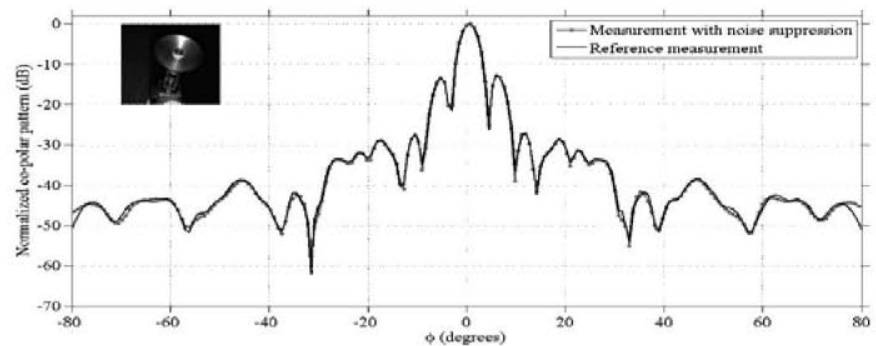

(c)

Fig. 10. Far-field comparison between (a) the reference pattern and the pattern with noise, (b) the reference pattern and the pattern after spatial filtering on the AUT aperture, and (c) the reference pattern and the pattern after spatial filtering on a larger surface in the horizontal plane.

to be concentrated on the AUT region. Nevertheless, this assumption is not completely correct. A small field contribution always exists outside the AUT. Due to this fact, when using a filter with the same size as the AUT aperture, a portion of desired signal is canceled. This cancellation introduces an error in the side-lobes of the reconstructed far-field pattern around $\pm 60^{\circ}$, as observed in Fig. 10(b). To avoid this negative effect, spatial filtering over a larger area must be employed to account for all of the desired data. However, this new filter integrates more noise power. Therefore, a compromise is required between the noise reduction and an accurate far-field representation. In this particular case, a filtering window with a size one and a half times the size of the antenna aperture was also employed. The improvement achieved with this filtering is $10.06 \mathrm{~dB}$, which is smaller than that one obtained with the filtering of minimum size. However, because more information about field radiated by the AUT is not filtered out, the reference pattern is better retrieved, as it is possible to see in Fig. 10(c).

\section{CONClusion}

Two simple and efficient methods to improve the signal-to-noise ratio in the far-field results obtained from cylindrical near-field measurements were presented. The first method applies a filtering in the modal domain and its main drawback is the necessity of oversampling, increasing the measurement time. However, this is not a critical aspect when measuring in a multi-probe system. Moreover, it is possible to improve the signal-to-noise ratio in all directions. The second method employs a source reconstruction technique to filter out the noise in the extreme near-field. Unlike the modal filtering method, this second approach does not require an oversampling. In addition, a better improvement is obtained using less measured data because of the use of more a priori information about the AUT. Whereas in the modal filtering, only information about the size of the AUT is required to defined the visible region, in the spatial filtering method both the size and the exact position of the antenna need to be known. Moreover, the spatial filtering method only provides a noise reduction in the forward hemisphere and it was noted that works better for planar aperture antennas because the region, in which the fields are assumed to be concentrated, is well defined. In contrast, the modal filtering method is independent of the shape of the antenna and it can be applied to antennas with arbitrary geometries obtaining similar improvements. Approximate theoretical expressions were derived for the improvement achieved with both methods. Finally, these expressions as well as the effectiveness of the methods were verified through application to both simulated and measured near-field data.

\section{REFERENCES}

[1] A. D. Yaghjian, "An overview of near-field antenna measurements," IEEE Trans. Antennas Propag., vol. AP-34, no. 1, pp. 30-44, Jan. 1986.

[2] R. C. Johnson, H. A. Ecker, and J. S. Hollis, "Determination of far-field antenna patterns from near-field measurements," Proc. IEEE, vol. 61, no. 12 , pp. 1668-1694, Dec. 1973.

[3] P. Petre and T. K. Sarkar, "Planar near-field to far-field transformation using an equivalent magnetic current approach," IEEE Trans. Antennas Propag., vol. 40, no. 11, pp. 1348-1356, Nov. 1992.

[4] A. C. Newell, "Error analysis techniques for planar near-field measurements," IEEE Trans. Antennas Propag., vol. 36, no. 6, pp. 754-768, Jun. 1988.

[5] L. A. Muth, "Displacement errors in antenna near-field measurements and their effect on the far-field," IEEE Trans. Antennas Propag., vol. 36, no. 5, pp. 581-591, May 1988.

[6] J. E. Hansen, "Error analysis of spherical near-field measurements," in Spherical Near-Field Antenna Measurements. London, U.K.: Peter Peregrinus, 1988, ch. 6, pp. 216-254.

[7] A. C. Newell and C. F. Stubenrauch, "Effect of random errors in planar near-field measurement," IEEE Trans. Antennas Propag., vol. 36, no. 6, pp. 769-773, Jun. 1988.

[8] J. B. Hoffman and K. R. Grimm, "Far-field uncertainty due to random near-field measurement error," IEEE Trans. Antennas Propag., vol. 36, no. 6, pp. $774-780$, Jun. 1988.

[9] J. Romeu, L. Jofre, and A. Cardama, "Far-field errors due to random noise in cylindrical near-field measurements," IEEE Trans. Antennas Propag., vol. 40, no. 1, pp. 79-84, Jan. 1992.

[10] J. Romeu and L. Jofre, "Effect of random errors in cylindrical near-field measurements," in Proc. IEEE/AP-S Int. Symp., London, ON, Canada, Jun. 24-28, 1991, pp. 1450-1453.

[11] P. Koivisto, "Reduction of errors in antenna radiation patterns using optimally truncated spherical wave expansion," Progress In Electromagn. Res., vol. PIER-47, pp. 313-333, 2004.

[12] L. J. Foged and M. Faliero, "Random noise in spherical near-field systems," in Proc. Antenna Meas. Tech. Assoc., AMTA, Salt Lake City, UT, Nov. 1-6, 2009, pp. 135-138.

[13] F. J. Cano-Fácila, S. Burgos, and M. Sierra-Castañer, "Novel method to improve the signal-to-noise ratio in the far-field results obtained from planar near-field measurements," IEEE Antennas Propag. Mag., vol. 52, no. 2, pp. 215-220, Apr. 2011.

[14] O. M. Bucci and C. Gennarelli, "Use of sampling expansions in nearfield-far-field transformations: The cylindrical case," IEEE Trans. Antennas Propag., vol. 36, no. 6, pp. 830-835, Jun. 1988. 
[15] A. D. Yaghjian, "Near-field antenna measurements on a cylinder surface: A source scattering matrix formulation," Nat. Bur. Stand. NBS Tech. Note 696, July 1977.

[16] S. Gregson, A. Newell, and G. Hindman, "Reflection suppression in cylindrical near-field antenna measurement systems-cylindrical MARS," in Proc. Antenna Meas. Tech. Assoc., AMTA, Salt Lake City, UT, Nov. 2009, pp. 119-125.

[17] G. E. Hindman and A. Newell, "Reflection suppression in a large spherical near-field range," in Proc. Antenna Meas. Tech. Assoc., AMTA, Newport, RI, Oct. 2005.

[18] D. W. Hess, "The IsoFilter ${ }^{\mathrm{TM}}$ technique: Extension to transverse offsets," in Proc. Antenna Meas. Tech. Assoc., AMTA, Austin, TX, Oct. 2006.

[19] S. Gregson, A. Newell, G. E. Hindman, and M. J. Carey, "Extension of the mathematical absorber reflection suppression technique to the planar near-field geometry," in Proc. Antenna Meas. Tech. Assoc., AMTA, Atlanta, GA, Oct. 2010.

[20] S. Gregson, B. M. Williams, G. F. Masters, A. Newell, and G. E. Hindman, "Application of mathematical absorber reflection suppression to direct far-field antenna range measurements," in Proc. Antenna Meas. Tech. Assoc., AMTA, Denver, CO, Oct. 2011.

[21] O. M. Bucci, G. D'Elia, and M. D. Migliore, "A general and effective clutter filtering strategy in near-field antenna measurements, "IEE Proc. Microw., Antennas, Propag., vol. 151, no. 3, pp. 227-235, Jun. 2004.

[22] W. M. Leach and D. T. Paris, "Probe compensated near-field measurements on a cylinder," IEEE Trans. Antennas Propag., vol. AP-21, no. 4, pp. 435-445, Jul. 1973

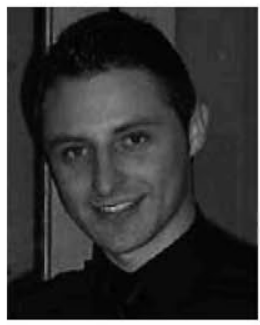

Francisco José Cano-Fácila was born in Don Benito, Spain, in 1985. He received the M.Sc. degree in telecommunication engineering and the Ph.D. degree from the Technical University of Madrid, Madrid, Spain, in 2008 and 2012, respectively.

In 2007, he worked for Telefónica Research and Development as part of the Final Client Services Department. Since 2008, he has been a Ph.D. student in the Radiation Group of the Signals, Systems and Radiocommunications Department, Technical University of Madrid (UPM). His main research interest is antenna measurement, focusing specifically on diagnostics techniques and nearfield to far-field transformations.

Dr. Cano-Fácila was awarded the highest marks for the telecommunication engineering degree in 2007. He obtained his M.Sc. degree with special distinction and was awarded a Ph.D. scholarship from the Spanish government. He also obtained the first place, AMTA student paper contest, for the paper titled "Novel method to improve the signal to noise ratio in the far-field results obtained from planar near-field measurements" presented in Atlanta in October 2010.

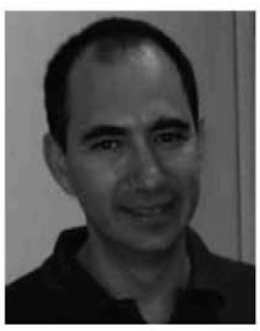

Manuel Sierra-Castañer (S'95-M'01) was born in Zaragoza, Spain, in 1970 . He received the degree in telecommunication engineering and the $\mathrm{Ph} . \mathrm{D}$. degree from the Technical University of Madrid (UPM), Madrid, Spain, in 1994 and 2000, respectively.

He worked for Airtel, a cellular company, from 1995 to 1997. Since 1997, he has worked in the University "Alfonso X" as an Assistant Professor. Since 1998, he has worked at the Technical University of Madrid as a Research Assistant, Assistant Professor, and Associate Professor. His current research interests involve planar antennas and antenna measurement systems.

Dr. Sierra-Castañer obtained the IEEE APS 2007 Schelkunoff Prize for his paper "Dual-Polarization Dual-Coverage Reflectarray for Space Applications" in 2007. 